\title{
Introdução a Supercondutores Granulares Artificiais: Redes de Junções Josephson
}

\author{
Introduction to artificial superconducting systems: Josephson junction arrays \\ F. M. Araújo-Moreira, W. Maluf, G. M. Cecato, e L. F. Kawashita \\ Grupo de Materiais e Sensores, Departamento de Física, \\ Centro Multidisciplinar de Desenvolvimento de Materiais Cerâmicos - CMDMC, \\ Universidade Federal de São Carlos, Caixa Postal 676, São Carlos, SP, 13565-905, Brasil \\ P. Barbara \\ Physics Department, Georgetown University \\ Washington DC, 2005\%-1228, USA \\ A. B. Cawthorne \\ Neocera, Inc., Beltsville MD, USA \\ A. Nielsen e C. J. Lobb \\ Center for Superconductivity Research, Department of Physics, \\ University of Maryland, College Park MD, 20742-4111 USA
}

Recebido em 23 de julho, 2002. Aceito em 19 de setembro, 2002.

\begin{abstract}
Redes de junções Josephson são dispositivos supercondutores com importantes aplicações tanto em tecnologia quanto em ciência básica. Neste artigo mostramos como estes dispositivos funcionam, suas propriedades magnéticas e de transporte, e como através das quais é possível estudar propriedades ainda controversas dos sistemas supercondutores granulares tais como o Efeito Meissner Paramagnético (PME), a Avalanche de Vórtices (VA) e outros efeitos relacionados com a granularidade.

Arrays of Josephson junctions are powerful superconducting devices with both technological and basic applications. In this review article, we describe how these devices work as well as their transport and magnetic properties. We also show how they can be used to explain some of the controversial properties of granular superconducting systems, like Paramagnetic Meissner Effect (PME), Vortex Avalanche (VA) and other granularity-related effects.
\end{abstract}

\section{Introdução}

$\mathrm{Na}$ atualidade, uma das áreas de maior desenvolvimento que utiliza materiais supercondutores é aquela relacionada com o projeto, a construção e a caracterização de dispositivos nanoestruturais visando a fabricação de sensores e detetores. Nesse desafio tecnológico tanto a ciência quanto a engenharia dos materiais utilizadas no processo são de crucial importância. Um grande número de aplicações tecnológicas de baixo custo seria viável se dispuséssemos de fontes de radiação eletromagnética compactas, sintonizáveis e efi- cientes, na faixa de frequêencias de $100 \mathrm{GHz}-1 \mathrm{THz}$. Além da vantagem óbvia de estender o espectro de freqüências disponíveis tecnologicamente, existem importantes aplicações potenciais, como por exemplo dispositivos que permitem a comunicação segura satélitesatélite (através da escolha de uma freqüência fortemente absorvida pela atmosfera), dispositivos sem fio para comunicação em alta velocidade, fontes de radiação para caracterizar sensores e para espectroscopia de materiais eletrônicos e osciladores locais para receptores em circuitos integrados do tipo single-chip.

\footnotetext{
*Autor para correspondência; Fax: (16) 261-4835; endereço eletrônico: faraujo@df.ufscar.br
} 
Fontes de corrente no limite inferior de freqüências incluem diodos dos tipos Gunn e IMPATT. Entretanto estes dispositivos apresentam características muito pobres em temos de eficiência e sintonia. Por outro lado, são disponíveis fontes de laser no limite superior de freqüências, mas estas fontes não são sintonizáveis. Em geral são muito grandes e ainda a potência de saída é baixa. Assim, não existem ainda dispositivos adequados para as aplicações mencionadas, e por isso estruturas baseadas em junções Josephson (JJ) são boas candidatas para atuarem como osciladores na faixa de freqüências proposta. A principal característica destas estruturas visando aplicações tecnológicas é o fato de que, quando uma voltagem constante $\mathrm{V}_{D C}$ é aplicada a uma junção Josephson, aparece uma corrente que oscila a uma freqüência $\nu$ da forma

$$
\nu=\frac{2 e}{h} V_{D C}=483 . V_{D C} \frac{\mathrm{GHz}}{\mathrm{mV}},
$$

onde $h$ é a constante de Planck e $e$ é a carga do elétron. Redes de junções Josephson (JJA) também têm sido extensivamente utilizadas em Física básica no estudo de transições de fase em sistemas bidimensionais [1]. Dentre estes, um dos mais estudados têm sido o sistema dos materiais supercondutores [2], dada a analogia que ambos apresentam. Neste trabalho mostramos alguns exemplos de propriedades magnéticas de JJA que visam a entender propriedades ainda controversas de sistemas supercondutores granulares, tanto convencionais (LTS) quanto de alta temperatura crítica (HTS).

\section{Supercondutividade e Junções Josephson}

A interpretação da supercondutividade como sendo um fenômeno quântico macroscópico, foi introduzida por $\mathrm{F}$. London em 1935. A teoria de Ginzburg-Landau (GLT) formulada em 1950 forneceu um enorme volume de informação em relação aos mecanismos responsáveis pela supercondutividade. Esta teoria teve como principal ponto a modificação da teoria de London (LT), e se baseou na introdução de um parâmetro dependente da posição $\Psi$, que seria uma medida da ordem (parâmetro de ordem) da fase supercondutora. A diferença da teoria prévia (teoria do dois fluídos, TFM), proposta por Casimir e Gorter em 1934, é que o parâmetro de ordem introduzido por Ginzburg e Landau é complexo, e pode ser considerado como uma função de onda associada aos portadores supercondutores (chamados superelétrons ou também pares de Cooper). Gorkov mostrou em 1959 que $\Psi$ é proporcional ao valor do gap de energia $\Delta$. A teoria BCS (1957) introduz uma escala de energias, onde os dois elétrons participantes do par de Cooper estão ligados com uma energia igual a $2 \Delta$, tipicamente da ordem de $10^{-3} \mathrm{eV}$ para supercondutores convencionais $\left(\mathrm{T}_{C} \leq 25 \mathrm{~K}\right)$. Assim, uma única função de onda é associada com um número macroscópico de elétrons que consideramos condensados no mesmo estado quântico. Isto significa que o estado supercondutor pode ser visto como um estado quântico macroscópico. Dessa maneira estaremos trabalhando com partículas (os superelétrons) que possuem carga e massa efetivas $m^{*}$ e $e^{*}$, que podem ser descritas como um todo por uma função de onda macroscópica dada por

$$
\psi=\rho^{1 / 2} \exp (i \phi),
$$

em que $\phi$ é a fase comum para todas as partículas e $\rho$ representa a densidade do macro-estado $|x\rangle$

$$
\langle s|\psi * \psi| s\rangle=|\psi|^{2}=\rho|s\rangle,
$$

A densidade de corrente elétrica pode ser escrita, na presença de um campo magnético, como

$$
\vec{J}=\frac{e *}{m *}\left[\frac{i \hbar}{2}(\psi \nabla \psi *-\psi * \nabla \psi)-\frac{e *}{c} \vec{A}|\psi|^{2}\right]
$$

em que $c$ é a velocidade da luz. Da teoria BCS (1957) temos que $m^{*}=2 m$ e $e^{*}=2 e$ (onde $m$ e $e$ são a massa e a carga eletrônicas, respectivamente), o que leva a

$$
\vec{J}=\rho \frac{e}{m}\left[\hbar \nabla \phi-\frac{2 e}{c} \vec{A}\right] .
$$

A invariância de calibre requer que, sob qualquer transformação do potencial vetor $A$ e do potencial escalar $U$, os observáveis físicos devem permanecer invariáveis

$$
\begin{aligned}
& \vec{A} \rightarrow \vec{A}+\nabla \chi \\
& \vec{U} \rightarrow \vec{U}-\frac{\partial \chi}{\partial t} .
\end{aligned}
$$

Isto significa que é válida a transformação de fase

$$
\phi \rightarrow \phi+\frac{2 e}{\hbar c} \chi
$$

A escolha dos valores constantes do escalar $\chi$ não afeta os valores do potencial, mas apenas os valores do fator de fase. Arbitrariamente, podemos dar valores para fase num determinado ponto, mas isto significará que a fase de todos os outros pontos esteja automaticamente determinada (ordenamento de longo alcance dos valores da fase). Obviamente, variações espaciais do valor da fase $\phi$, descrevem diferentes estados de corrente de transporte no supercondutor. Para um sistema em equilíbrio a invariância de calibre leva necessariamente a que $\Psi$ seja dependente do tempo. A evolução temporal de $\Psi$ em condições estacionárias obedece a equação usual dada por

$$
i h \frac{\partial \psi}{\partial t}=E \psi \text {. }
$$

Como pode ser observado da teoria microscópica de Gorkov (1959), a quantidade $E$ é equivalente a duas 
vezes o valor do potencial eletroquímico $\mu$. Este valor representa o mínimo de energia requerida para adicionar mais um par de Cooper ao sistema. Assim, podemos escrever

$$
\psi(r, t)=\psi(r) \exp \left[\frac{-2 i \mu t}{\hbar}\right]
$$

Consideramos dois supercondutores $S_{L}$ e $S_{R}$ separados por uma distância macroscópica. Nesta situação, a fase dos supercondutores pode mudar independentemente. Na medida em que ambos são colocados mais e mais próximos, e quando a separação entre eles é da ordem de algumas dezenas de Angstrons (30 $\AA$ ), observase a circulação de quase-partículas de um a outro supercondutor (single electron tunneling). Reduzindo ainda mais a separação entre $S_{L}$ e $S_{R}$, para distâncias tipicamente menores do que $10 \AA$, também circularão entre eles pares de Cooper. Este fenômeno de tunelamento de pares de Cooper entre os dois materiais supercondutores através de uma fina barreira isolante $(I)$ é denominado tunelamento Josephson. Neste caso, as fases entre ambos supercondutores não podem mais ser consideradas independentes uma da outra, e o sistema total $S_{L^{-}}$ $I-S_{R}$ pode ser considerado como um único supercondutor. Este fenômeno é conhecido como supercondutividade fraca em função dos baixos valores envolvidos dos parâmetros críticos. A teoria de Josephson está ligada diretamente a sistemas supercondutores fracamente acoplados. Assim, o sistema formado por dois supercondutores fracamente acoplados através de uma fina película isolante é chamado junção Josephson. Em 1962 , B. D. Josephson previu que seria possível o tunelamento de pares de Cooper neste tipo de estruturas, e que o mesmo poderia ser modelado através de certas equações. Estas equações relacionam a corrente de tunelamento $I_{S}$ com a voltagem $V$ através da junção via a diferença de fase $\delta \phi$ das funções de onda macroscópicas que descrevem ambos supercondutores:

$$
\begin{aligned}
& I_{S}=I_{0} \sin \delta \phi, \\
& \frac{d \delta \phi}{d t}=\frac{2 \pi}{\Phi_{0}} V .
\end{aligned}
$$

Nestas equações $I_{0}$ é uma constante correspondente à máxima corrente ou corrente crítica, que depende das características dos supercondutores que formam a junção, e $\Phi_{0}$ é o quantum de fluxo,

$$
\Phi_{0}=\frac{h c}{2 e}=2.07 \times 10^{-7} \text { gauss } \mathrm{cm}^{2}
$$

A Fig. 1 mostra a seção reta de uma junção Josephson. Os dois materiais supercondutores que formam a junção estão separados por uma barreira isolante de espessura $2 a$. Cada supercondutor tem espessura $\left(b_{1} \mathrm{e}\right.$ $b_{2}$ ) muito maior do que as respectivas profundidades de penetração $\lambda_{1}$ e $\lambda_{2}$.

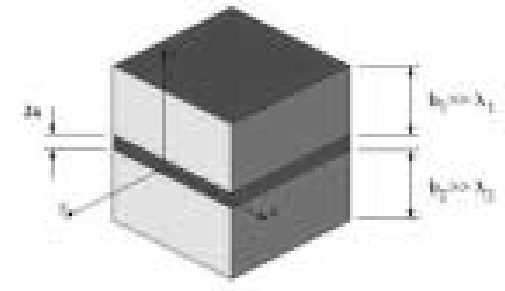

Figura 1. Seção reta de uma junção Josephson.

As relações de Josephson são locais e geralmente $\delta \phi \neq 0=$ constante. Uma corrente finita com valor máximo igual a $I_{0}$ poderá fluir através da barreira, mas com $V=0$ (efeito Josephson DC). O efeito Josephson foi observado pela primeira vez em 1963 por Anderson e Rowell.

A corrente de tunelamento contém um termo linear correspondente de quase-partículas $\left[\sigma_{0} \mathrm{~V}\right]$ e um termo $\left[\sigma_{1} \mathrm{~V} \cos \delta \phi\right]$ relacionado com os efeitos de interferência entre quase-partículas e pares de Cooper que pode ser escrita como

$$
I=I_{0} \sin \delta \phi+\left[\sigma_{0}(V, T)+\sigma_{1}(V, T) \cos \delta \phi\right] V .
$$

Neste caso $\sigma_{0}$ e $\sigma_{1}$ são a parte real e a parte imaginária da condutividade complexa, respectivamente $\left(\sigma=\sigma_{0}\right.$ $\left.+\mathrm{i} \sigma_{1}\right)$. Para valores de corrente de transporte menores do que a corrente crítica, a voltagem através da junção é zero, como predito pelo efeito Josephson DC. Quando a corrente ultrapassa o valor $I_{0}$ a voltagem da junção passa para o valor $V_{g}$, (voltagem do gap) que depende da energia do gap $\Delta$ de cada supercondutor de acordo com a relação

$$
V_{g}=\frac{\Delta_{1}-\Delta_{2}}{e}
$$

Para valores $V>V_{g}$, a corrente é transportada principalmente por quase-partículas e a parte correspondente na curva $I \times V$ é descrita pela resistência do estado normal.

Existem diferentes maneiras de produzir uma junção fraca (weak link) entre dois supercondutores (Fig. 2). Como mencionado anteriormente, um tipo de junção fraca é obtido "sanduichando" uma fina película isolante entre dois materiais supercondutores (tunnel junction). A barreira pode ser feita a partir da oxidação do filme da base ou pela deposição de camadas adicionais de um metal oxidado, de um semicondutor ou de um metal normal. Tipicamente, quando utilizado material isolante, a espessura da barreira é de alguns nanômetros. Para uma barreira feita de material semicondutor ou normal, ela possui espessura de 10 a 100 vezes maior. Outro exemplo de junção Josephson é a estrutura conhecida como microponte. Essa 
estrutura é constituída de um único filme supercondutor com um grande estreitamento, tipicamente menor que o comprimento de coerência supercondutor $\xi$, tal que o parâmetro de ordem é suprimido nessa região. $\mathrm{O}$ último exemplo de junção Josephson é aquele denominado de junção de contacto pontual.

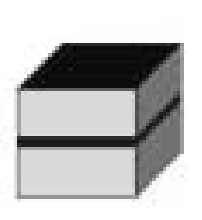

Japrom That

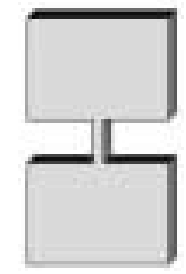

Aarbo Micropate

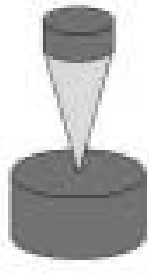

Jascas Pereo
Figura 2. Tipos de junções.

\section{Efeito do Campo Magnético numa junção Josephson}

Vejamos agora o efeito de um campo magnético $H$ aplicado à junção ao longo da direção y (Fig. 3). Para isso, tomando como base a equação [4] calculamos a diferença de fase entre dois pontos com coordenadas $x$ e $(x+d x)$

$$
\nabla \phi_{L, R}=\frac{2 e}{\hbar c}\left[\frac{m c}{2 e^{2} \rho} \vec{J}_{s}+\vec{A}\right]
$$

o que nos leva a

$$
\begin{aligned}
& \phi_{R a}(x)-\phi_{R b}(x+d x)=\frac{2 e}{\hbar c} \int_{C_{R}}\left(\vec{A}+\frac{m c}{2 e^{2} \rho} \vec{J}_{s}\right) \cdot d \vec{l}, \\
& \phi_{L b}(x+d x)-\phi_{L a}(x)=\frac{2 e}{\hbar c} \int_{C_{L}}\left(\vec{A}+\frac{m c}{2 e^{2} \rho} \vec{J}_{s}\right) \cdot d \vec{l},
\end{aligned}
$$

Admitindo que a espessura do filme supercondutor é muito maior que a profundidade de penetração de London, os contornos $C_{L}$ e $C_{R}$ podem ser estendidos fora da região de penetração onde a densidade de corrente de blindagem $J_{S}$ desaparece.

As porções de $C_{L}$ e $C_{R}$ na região de penetração podem ser escolhidas como sendo perpendiculares à $J_{S}$. A partir destas considerações, podemos desprezar os segundos termos nas integrais das Eqs, (16) e (17), e teremos que

$$
\phi(x+d x)-\phi(x)=\frac{2 e}{\hbar c}\left[\int_{C_{L}}(\vec{A} \cdot d \vec{l})+\int_{C_{R}}(\vec{A} \cdot d \vec{l})\right]
$$

Ainda, desprezando a espessura da barreira, temos que

$$
\phi(x+d x)-\phi(x)=\frac{2 e}{\hbar c} \oint(\vec{A} \cdot d \vec{l})
$$

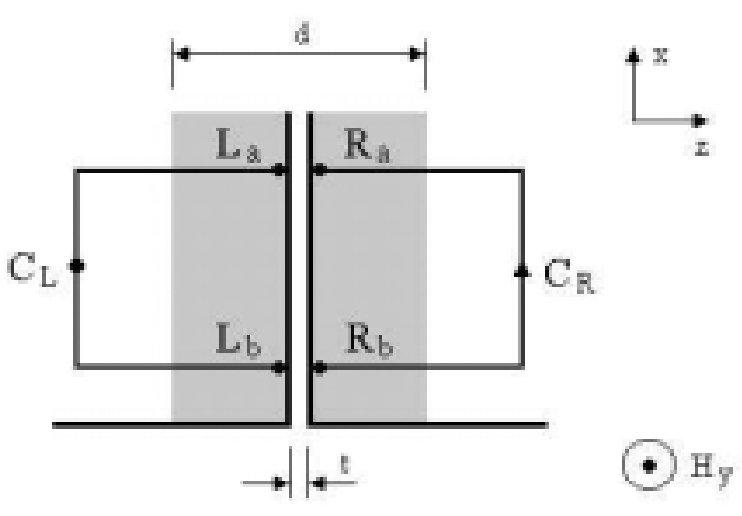

Figura 3. Campo aplicado à junção.

A integral de linha pode ser substituída pela integral de superfície do campo magnético

$$
\oint(\vec{A} \cdot d \vec{l})=H_{y}\left(\lambda_{R}+\lambda_{L}+t\right) d x
$$

Em termos diferenciais, temos que

$$
\frac{d \phi}{d x}=\frac{2 e H_{y}}{\hbar c}\left(\lambda_{R}+\lambda_{L}+t\right) d x,
$$

em que $\lambda_{R}, \lambda_{L}$ e $t$ são as profundidades de penetração de London e a espessura da barreira, respectivamente.

Integrando a Eq. (21), temos que

$$
\phi-\phi_{0}=D \frac{2 e}{\hbar c} H_{y} x .
$$

Nesta equação, definimos

$$
D=\lambda_{R}+\lambda_{L}+t
$$

como a penetração magnética. Então, das Eqs. (11) e (22), obtemos que

$$
J=J_{0} \sin \left[D \frac{2 e}{\hbar c} H_{y} x+\phi_{0}\right]
$$

Esta equação indica que a supercorrente de tunelamento é modulada espacialmente pelo campo magnético. Devido ao caráter periódico da Eq. (24), pode-se concluir imediatamente que existem condições para as quais a corrente de tunelamento líquida é nula. Por exemplo, uma junção retangular que apresenta uma distribuição de corrente de tunelamento uniforme para campo zero, exibirá uma dependência com o campo 
magnético aplicado similar ao clássico diagrama de Fraunhofer (Fig. 4).

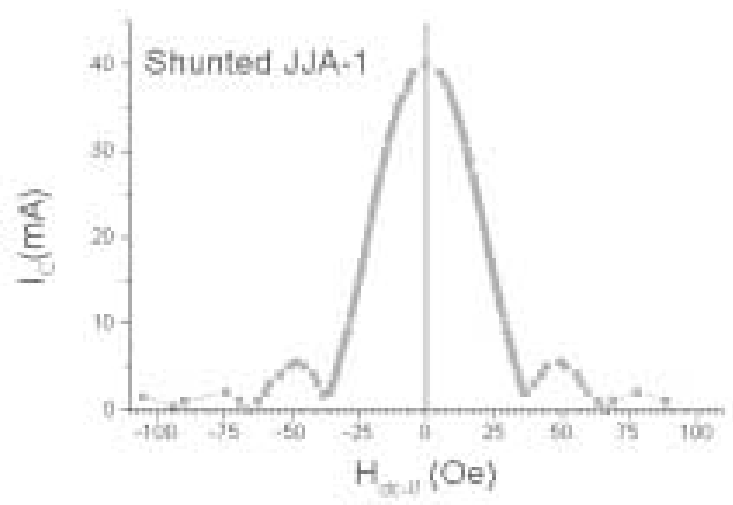

Figura 4. Diagrama de Fraunhofer.

A expressão analítica para $I(H)$ será então dada por

$$
I(H)=I_{0}\left|\frac{\sin \frac{\Phi}{\Phi_{0}} \pi}{\frac{\Phi}{\Phi_{0}} \pi}\right|,
$$

em que $\Phi=H L D$,. Isso significa que os valores dos pontos de mínimo na curva de difração de Fraunhofer, acontecem em valores múltiplos de $\Phi_{0}$.

\section{Degraus de Fisk}

É possível obter diferentes tipos de estados dinâmicos envolvendo a propagação de fluxóides em conjuntos de junções Josephson acopladas. Assim, distinguimos entre modos ressonantes (Zero field steps e Fiske steps), e o modo flux-flow unidireccional. Os modos ressonantes aparecem em junções supra-amortecidas onde o comprimento de decaimento das ondas refletidas nas junções depende do parâmetro de supra-amortecimento $\alpha$. O movimento dos fluxóides é governado pela corrente de transporte através das forças de Lorentz. Um fluxóide gera um pulso de energia eletromagnética quando atinge a fronteira da junção tal que a propagação periódica dos fluxóides produz a emissão de radiação.

$\mathrm{Na}$ ausência de campo magnético externo, a curva $I \times V$ apresenta uma série de ramificações (Fig. 5). Estas peculiaridades na curva são denominadas de (ZFS) e são numerados em ordem crescente a partir daquele que se apresenta na voltagem mais baixa. O estado dinâmico correspondente pode ser descrito em termos de um ou mais fluxóides propagando na junção e sendo refletidos como anti-fluxóides nas bordas.

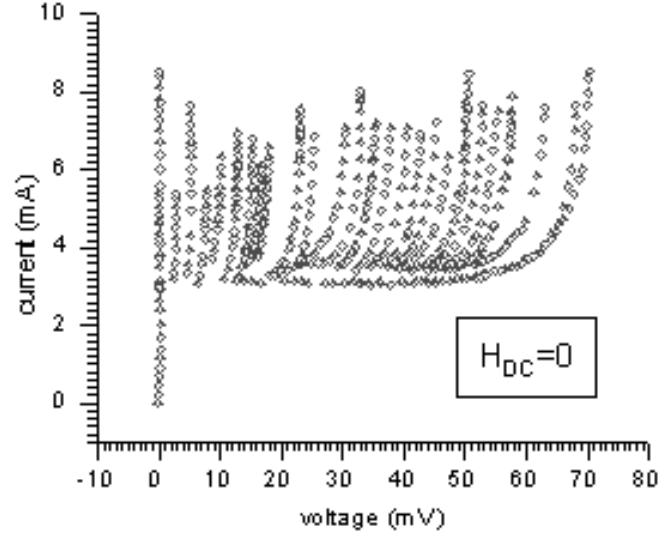

Figura 5. Ramificações da curva I x V.

Os anti-fluxóides propagam-se no sentido oposto, na direção da outra borda onde eles serão refletidos como fluxóides, e assim o mecanismo começa novamente. A voltagem DC correspondente a esse estado pode ser avaliada facilmente a partir das Eqs. (11) e (12), considerando que em cada reflexão a fase aumenta $4 \pi$, e que o tempo correspondente é dado por um período de oscilação dado por

$$
T=\frac{2 L}{u} .
$$

Nesta equação, $L$ é o comprimento da junção e $u$ é a velocidade do fluxóide. Para um número $n$ de fluxóides a voltagem DC associada é dada por

$$
V_{D C}^{n}=\frac{\hbar}{2 e} \frac{2 \pi u n}{L} .
$$

Aumentando a corrente de transporte através da junção aumentará a voltagem de cada degrau até o valor limite que corresponde a $u=\bar{c}$. Este estado gera a emissão de microondas numa freqüência $f=n u / 2 L$.

$\mathrm{Na}$ presença de um campo magnético aparecem nas curvas $\mathrm{I} \times \mathrm{V}$ os denominados degraus de Fiske (FS) na forma de ramificações com altura fortemente dependente da intensidade do campo aplicado. A dinâmica correspondente é explicada em termos da propagação de fluxóides seguidos pela reflexão de ondas de plasma geradas nas bordas e propagando-se em forma reversa na direção da outra borda onde novos fluxóides são criados. Neste caso, a voltagem DC é a metade da voltagem correspondente ao processo ZFS de mesma ordem. Os FS também aparecem em junções de comprimentos pequeno e médio. Neste caso, a largura dos fluxóides é comparável com o comprimento da junção. A dinâmica nesse estado está relacionada com a interação linear da corrente Josephson com os modos ressonantes da cavidade. O regime de flux-flow consiste no movimento unidireccional viscoso dos fluxóides que são nucleados num dos extremos da junção pelo campo magnético aplicado, 
formando uma rede de fluxóides Josephson propagando na junção. Este estado também aparece como um degrau na curva $I \times V($ FFS $)$ cuja voltagem é dada por

$$
V_{D C}=u H_{e} d,
$$

em que $d$ é a espessura magnética $\left(d=2 \lambda_{L}+t\right.$, onde $t$ é a espessura da barreira isolante e $\lambda_{L}$ é a profundidade de penetração.

\section{Redes de Junções Josephson}

Quando as junções descritas anteriormente são fabricadas formando um arranjo bidimensional ou tridimensional, então o conjunto forma uma rede de junções Josephson (JJA). Atualmente, as redes com maior número de aplicações tecnológicas são fabricadas com supercondutores convencionais, por exemplo nióbio e óxidos como o $\mathrm{AlO}_{x}$. Basicamente, JJA podem ser fabricadas em dois tipos de arranjos, denominados shunted e unshunted. A diferença básica entre ambos é a existência de uma resistência de molibdênio do tipo shunted em paralelo com a junção. A presença dessa resistência muda completamente o valor do parâmetro de Stewart-McCumber, $\beta_{C}$ (definido na Eq. 31). Na Fig. 6 mostramos o perfil de uma junção shunted de $\mathrm{Nb}-\mathrm{AlO}_{x}-\mathrm{Nb}$

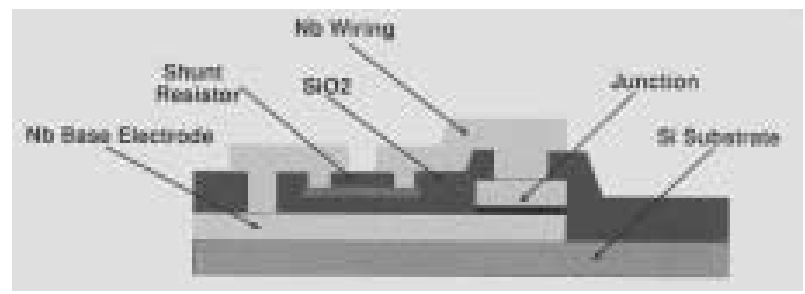

Figura 6. Perfil de uma junção shunted $\left(\mathrm{Nb}-\mathrm{AlO}_{x}-\mathrm{Nb}\right)$.

Os supercondutores sinterizados HTS são materiais granulares por natureza. Tipicamente, grãos supercondutores estão distribuídos aleatoriamente numa matriz formada por material intergranular com características e propriedades próprias (Fig. 7). Esta matriz intergranular tem sido freqüentemente associada com uma rede de junções Josephson (JJA).

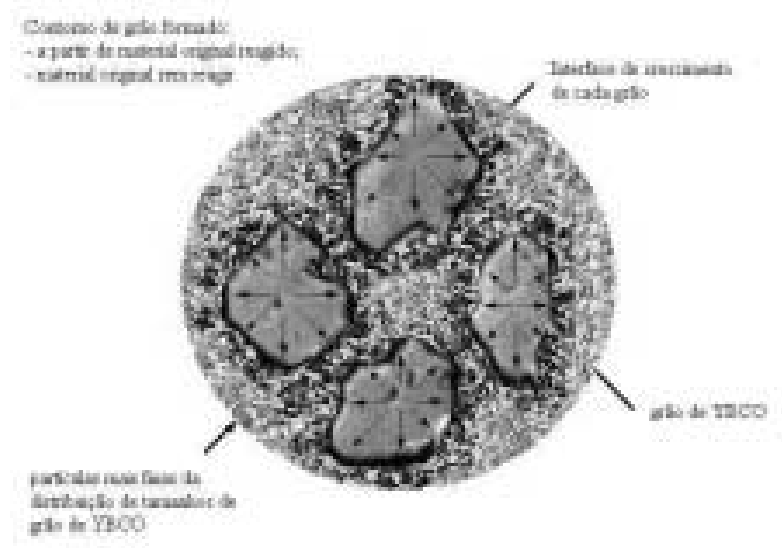

Figura 7. Matriz intergranular.

A analogia entre materiais granulares e JJA tem permitido compreender muitas das propriedades e mecanismos dos HTS. Por exemplo, as propriedades das JJA podem ser associadas diretamente com o modelo do estado crítico (CSM) [2] e com propriedades mais polêmicas como o Efeito Meissner Paramagnético (PME) [3-7] observado nos HTS.

Nas Figs. 8 e 9, mostramos a fotografia de uma região de uma rede formada por $100 \times 150$ junções, para um JJA do tipo unshunted e para um do tipo shunted respectivamente.

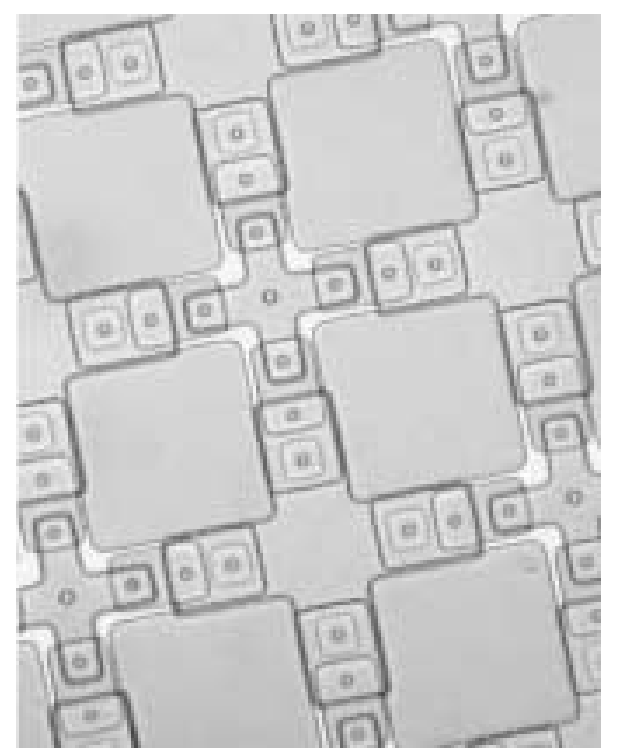

Figura 8. Região de um array $100 \times 150$ do tipo unshunted. 


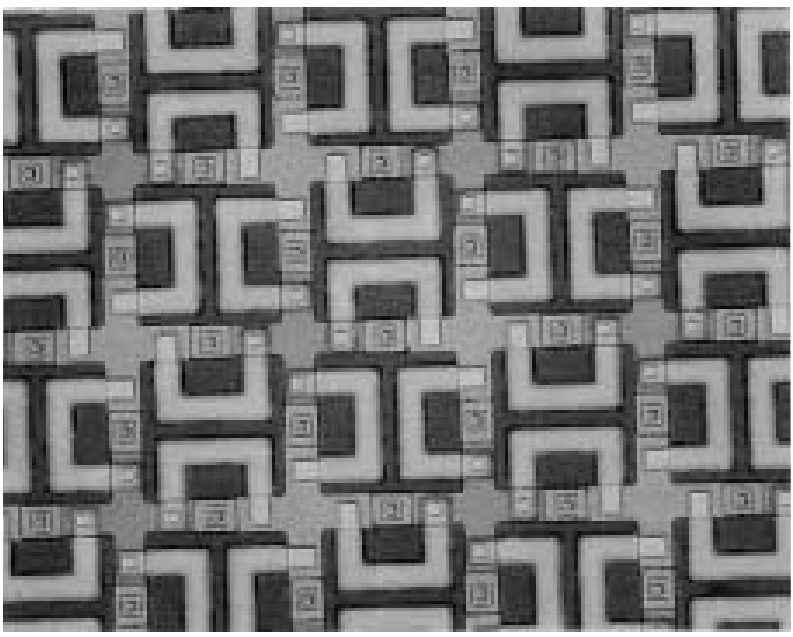

Figura 9. Região de um array $100 \times 150$ do tipo shunted.

\section{Remanência Magnética em Redes de Junções Josephson}

Para analisar os resultados experimentais de suscetibilidade magnética $\mathrm{AC} \chi_{A C}(\mathrm{~T})$, obtidos de JJA unshunted, podem ser realizadas simulações numéricas tomando como base apenas uma plaquette formada por um loop de quatro junções Josephson [4-6]. Para simular a resposta magnética dessa estrutura, devemos considerar a dependência com a temperatura da densidade de corrente crítica dada por

$$
J_{C}(t)=J_{C}(0)(1-t)^{1 / 2} \tanh \left\{\frac{1.54(1-t)^{1 / 2}}{t}\right\}
$$

em que $t=T / T_{c}$. Dessa maneira, mostramos que a resposta magnética de uma JJA pode ser adequadamente descrita pelo simples modelo de uma plaqueta contendo quatro JJ. Essa resposta é controlada pelos parâmetro:s

$$
\begin{gathered}
\beta_{L}(T)=\frac{2 \pi I_{C}(T) L}{\Phi_{0}}, \\
\beta_{C}(T)=\frac{2 \pi I_{C}(T) C_{J} R_{J}^{2}}{\Phi_{0}},
\end{gathered}
$$

em que $L$ é a indutância da plaqueta, $I_{C}(T)$ é corrente crítica dependente da temperatura, $C_{J}$ e $R_{J}$ são a capacitância e a resistência shunt, Quando ambos parâmetros são maiores do que 1, tipicamente da ordem de 10 ou ainda maiores, aparece um comportamento reentrante na suscetibilidade magnética que temos associado ao efeito Meissner paramagnético (PME) [4-6].

Seguindo a mesma linha de trabalho, simulamos curvas $\mathrm{M}(\mathrm{H})$ para o mesmo modelo mencionado anteriormente, baseado num loop contendo quatro JJ. Um exemplo dos resultados obtidos é mostrado na Fig. 10, onde utilizamos os parâmetros $\beta_{L}=\beta_{C}=30$, para três valores diferentes da temperatura. A partir da análise dessa figura, é evidente que se olharmos para a janela experimental correta (i.e., a temperatura) a JJA vai reter um certo momento magnético, $M_{r}$, após ter sido excitado por um campo magnético cujo valor de pico é maior do que um certo valor de corte, que depende da temperatura. Este comportamento, que pode ser razoavelmente visível para sistemas granulares supercondutores minimamente organizados, tem sido detectado em diversos sistemas tridimensionais de JJA, tanto em HTS quanto em LTS [3-5]. Assim, a ocorrência de remanência magnética é uma característica esperada em sistemas supercondutores granulares.

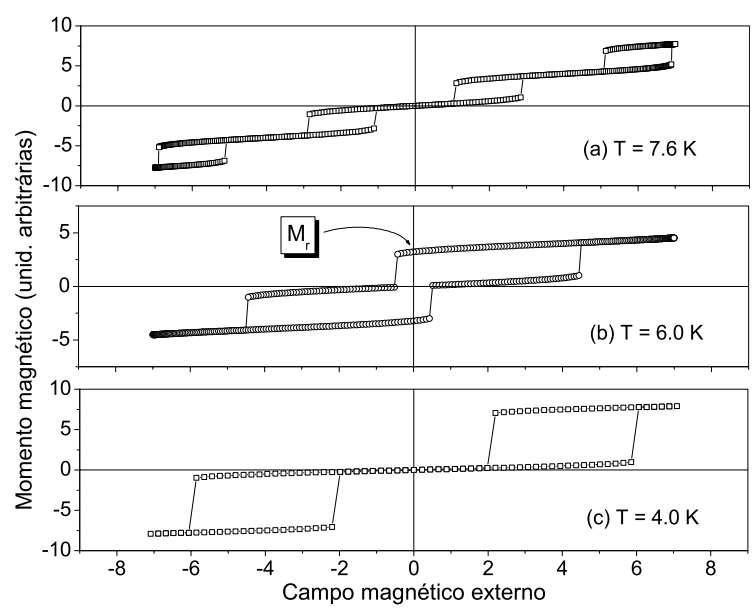

Figura 10. Simulação numérica $M(H)$ baseada num loop contendo quatro JJ, para $\beta_{L}=\beta_{C}=30$, para (a) $T=7.6$ $\mathrm{K}$, (b) $T=6.0 \mathrm{~K}$, and (c) $T=4.0 \mathrm{~K}$ a partir da análise desta figura, na janela experimental correta (i.e., a temperatura $T=6.0 \mathrm{~K})$ a JJA vai reter um certo momento magnético, $M_{r}$, após ter sido excitado por um campo magnético cujo valor de pico é maior do que um certo valor de corte, que depende da temperatura.

\section{Redes de Junções Josephson e Supercondutores Granula- res}

Como já mencionamos, sistemas granulares supercondutores podem ser visualizados como uma coleção de grãos supercondutores distribuídos numa matriz normal ou fracamente supercondutora. Por essa razão o termo granularidade está intimamente relacionado aos HTS. Através dele se justifica que as propriedades magnéticas e de transporte desses materiais se manifestam usualmente através de uma resposta que tem duas ou mais componentes. No caso de duas componentes, a primeira delas (em temperatura mais alta) 
representa a contribuição intragranular, que está associada às propriedades intrínsecas dos grãos supercondutores. A outra componente (em baixas temperaturas) se origina no material intergranular e está associada com a supercondutividade fraca, relacionada aos weak links. Neste caso, as propriedades intragranulares serão intrínsecas ao material, enquanto as intergranulares serão extrínsecas e gerarão efeitos que serão dependentes das condições de preparação do material. Isto tem permitido criar de maneira controlada defeitos nos HTS para estudar o aumento da densidade de corrente crítica, $J_{C}$, como conseqüência da criação de centros de ancoramento adicionais.

Dessa maneira, as junções (por exemplo, de Nb$\mathrm{AlO}_{x}-\mathrm{Nb}$ ) de uma JJA serão equivalentes ao material intergranular (weak link) e as ilhas de Nb serão equivalentes ao material intragranular dos HTS.

\section{Métodos Experimentais para Caracterizar JJA}

Existem vários caminhos para caracterizar redes de junções Josephson. Vamos descrever aqui as técnicas com as quais temos trabalhado diretamente. Essas consistem de curvas $I \times V$, microscopia de SQUID, diagrama de Fraunhofer $I_{C}\left(H_{D C}\right)$, e suscetibilidade magnética $\mathrm{AC}, \chi_{A C}$.

A caracterização através da suscetibilidade magnética, foi realizada utilizando a técnica $\mathrm{AC}$ convencional (bobina primária e duas bobinas secundárias), numa montagem especial para amostras na forma de filmes. Neste caso o método é conhecido como método de blindagem [7], e utilizamos a montagem em forma de reflexão, como descrito por Jeanneret et al.[8]. Assim, uma corrente alternada alimenta a bobina primária, e o sinal de saída das bobinas secundárias (ligadas entre si em oposição de fase), é detectado com um amplificador lock-in. Desta maneira, e após a correspondente separação das fases, medimos duas voltagens $V_{L}$ e $V_{R}$, proporcionais a $\chi^{\prime}$ e $\chi$ ", respectivamente. O campo magnético externo $\mathrm{H}_{D C}$ é mantido paralelo ao plano da amostra, e perpendicular ao campo $\mathrm{h}_{A C}$ originado na bobina primária, como mostrado na Fig. 11.

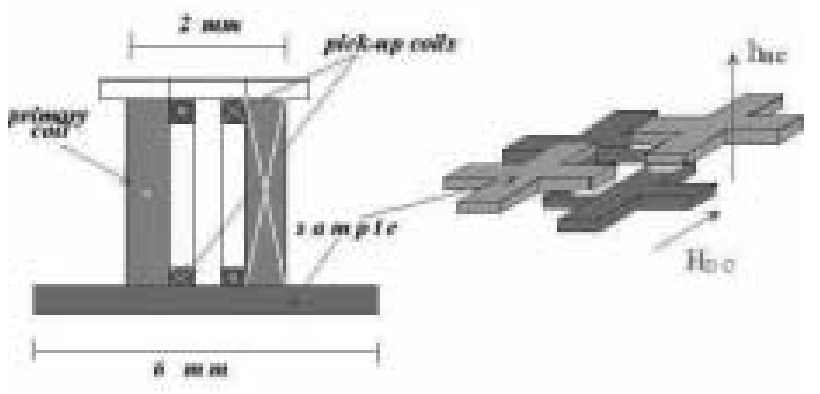

Figura 11. Montagem experimental screenning method.
Na Fig. 12, mostramos a componente $\chi^{\prime}$ da curva de suscetibilidade magnética AC vs. temperatura, para uma amostra JJA-unshunted. Nesta medida observamos os degraus que estariam relacionados com o fenômeno de avalanche de fluxo, observado em HTS $[9,10]$. Ainda na mesma figura, amplificamos a região dos degraus, mostrando que eles aparecem apenas na região de temperaturas correspondentes após o mínimo na reentrância. Quando estes degraus são suficientemente amplificados, vemos que também parecem ser reentrantes na temperatura. Eles somente aparecem nas amostras do tipo unshunted, o que parece estar associado ao parâmetro de McCumber $\left(\beta_{C}\right)$ [11].

$\mathrm{Na}$ caracterização magnética das amostras também determinamos o diagrama de Fraunhofer, mostrado anteriormente na Fig. 4. Assim, através da técnica usual para determinação de uma curva $I \times V$, encontramos a dependência da corrente crítica $I_{C}$ de cada rede shunted e unshunted, como função do campo magnético externo aplicado $H_{D C}$. A partir do esquema experimental mostrado na Fig. 11 vemos que $H_{D C}$ é paralelo ao plano da amostra, ou seja, vai atuar apenas sobre a corrente crítica das junções que formam a rede, praticamente não induzindo fluxo magnético dentro das plaquetes formadas por loops de quatro junções. De acordo com nossos cálculos, o desvio do plano da amostra em relação a $H_{D C}$ é de apenas $0.1^{\circ}$, o que significa menos de $1 \mathrm{mOe}$ de fluxo magnético induzido na região de cada loop da rede.

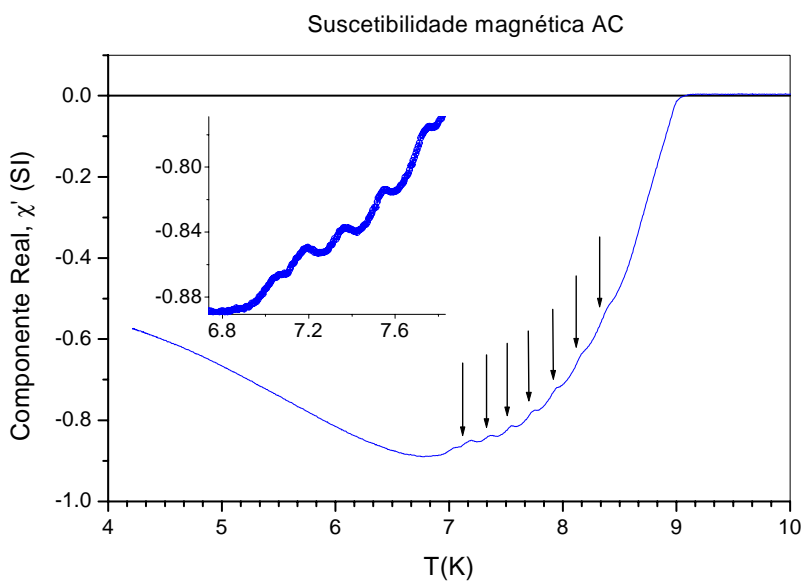

Figura 12. Componente $\chi^{\prime}$ da curva de suscetibilidade magnética AC para uma amostra unshunted. Na inserção mostramos a amplificação da região dos degraus, onde aparecem apenas para temperaturas correspondentes após o mínimo na reentrância.

\section{Sumário}

Neste artigo abordamos os conceitos básicos da Física associada às redes de junções Josephson. Sem 
dúvida que, na atualidade, a área de desenvolvimento de dispositivos supercondutores nanoestruturais é uma das mais promissoras dentro daquelas que visam a fabricação de sensores e detetores para diversos tipos de aplicações. Por outro lado, do ponto de vista de ciência básica, o estudo de redes de junções Josephson, através da equivalência em muitos aspectos com sistemas supercondutores granulares, permite o entendimento de muitas propriedades básicas ainda controversas desses sistemas, como por exemplo o efeito Meissner paramagnético (PME) ou o fenômeno de avalanche de vórtices (VA), observados nos HTS. Assim, o estudo destas estruturas, cuja grande vantagem é a de serem completamente controláveis em termos de parâmetros de fabricação, abrange problemas básicos e de aplicações. Dessa maneira, seu enorme potencial em ambas áreas as tornam elementos importantes em ciência e tecnologia.

\section{Agradecimentos}

Agradecemos à FAPESP, CNPq, National Science Foundation (NSF) e United State Air Force Office of Scientific Research (USAF-OSR) pelo suporte financeiro à pesquisa das propriedades de redes de junções Josephson.

\section{Referências}

[1] Ch. Leemann, Ph. Lerch, G. A. Racine, e P. Martinoli, Phys. Rev. Lett. 56, 1291 (1986); Physics Today,
Special Issue: Superconductivity (1986); Physics Today, Special Issue: High-Temperature, Superconductivity (1991).

[2] D. X. Chen and A. Sánchez, J. Appl. Phys. 70, 5463 (1991).

[3] F. M. Araújo-Moreira, A. Cawthorne, A. P. Nielsen, P. Barbara e C. J. Lobb (submetido Phys. Rev. B, 2002).

[4] F. M. Araújo-Moreira, P. Barbara, A. Cawthorne e C. J. Lobb; Phys. Rev. Lett. 78, 4625 (1997).

[5] F. M. Araújo-Moreira, P. Barbara, A. Cawthorne e C. J. Lobb; Bull. of the Am. Phys. Soc. 42, 185 (1997).

[6] P. Barbara, F. M. Araújo-Moreira, A. B. Cawthorne, e C. J. Lobb; Phys. Rev. B 60, 7489 (1999).

[7] J. H. Claassen, em Magnetic Susceptibility of Superconductors and Other Spin Systems; p.405, Ed. By R. H. Hein et al.; Plenum Press, New York (1991).

[8] J. L. Jeanneret, G. A. Gavilano, A. Racine, Ch. Leemann, and P. Martinoli, Appl. Phys. Lett. 55, 2336 (1989).

[9] F. M. Araújo-Moreira, P. Barbara, W. Maluf, and C. J. Lobb (submetido ao Phys. Rev. Lett. 2002).

[10] W. Maluf and F. M. Araújo-Moreira, Braz. J. of Physics, 32, 717 (2002).

[11] F. M. Araújo-Moreira, P. Barbara, W. Maluf, and C. J. Lobb (submetido ao Physica C, 2002). 